\title{
METHODS TO STUDY THE DYNAMICS OF THE OORT CLOUD COMETS II: MODELLING THE GALACTIC TIDE
}

\author{
Marc Fouchard ${ }^{1,2,5}$, Christiane Froeschlé ${ }^{3}$, Sławomir Breiter ${ }^{4}$, Roman \\ Ratajczak $^{4}$, Giovanni B. Valsecchi ${ }^{5}$, and Hans Rickman ${ }^{6}$ \\ 1 Laboratoire d'Astronomie de Lille/Université de Lille, \\ 1 impasse de l'Observatoire 59000 Lille, France \\ 2 Institut de Mécanique Céleste et de Calcul d'Ephémérides, UMR 8028 du CNRS \\ email: fouchard@imcce.fr \\ 3 Observatoire de la Côte d'Azur, BP 4229, FR-06304 Nice, France \\ email: froesch@obs-nice.fr \\ 4 Astronomical Observatory of A. Mickiewicz University, \\ Słoneczna 36, Poznań, 60-286, Poland \\ email: breiter@amu.edu.pl, astromek@amu.edu.pl \\ 5 INAF-IASF, Via Fosso dell Cavaliere 100, 00133 Roma, Italy \\ email: giovanni@iasf-roma.inaf.it \\ 6 Uppsala Astronomical Observatory, Box 515, SE-75120 Uppsala, Sweden \\ email: hans@astro.uu.se
}

\section{Introduction}

The present paper follows the accompanying Paper I, in which we investigate different algorithms to compute the orbital perturbations of Oort cloud comets caused by passing stars. In the present one, Paper II, we present several fast integrators and mappings which modelize the effects of the galactic tide. The results computed with these methods are compared with those obtained using a numerical integration of the equations of motion.

To model the normal and radial components, with respect to the galactic plane, of the Galactic tide different methods and mappings are developed in Section 2. First of all in Section 2.1 we give the equations of motion of a comet perturbed by the Galactic tide. Then the following models are described, namely:

- a symplectic and regularised integrator (Section 2.2);

- some averaged Hamiltonian models using, according to the value of the orbital eccentricity $e$, either the Delaunay elements or the "Matese elements" and, for each model, mappings based on the respective Taylor development (Section 2.3); 
- a Lie-Poisson averaged model (Section 2.4).

In Section 3 the results of calculations performed using the different models are compared to those obtained by numerical integrations. This comparison allows us to build models which use a composition of two different kinds of integrators in order to increase the velocity of the integrations minimising the loss of accuracy (Section 4). Section 5 is devoted to the conclusions.

\section{Models of Galactic tide effects on cometary orbits}

\subsection{The Cartesian model}

To establish the equations of motion, we consider two different heliocentric frames. The first one is a rotating frame with the $\hat{x}^{\prime}$-axis in the radial direction pointing towards the Galactic center, the $\hat{y}^{\prime}$ axis pointing transversely along the local circular velocity, and $\hat{z}$ completing a right-handed system. The fixed frame $(\hat{x}, \hat{y}, \hat{z})$ is such that it coincides with the rotating frame $\left(\hat{x}^{\prime}, \hat{y}^{\prime}, \hat{z}\right)$ at time $t=0$ while keeping its axial directions fixed (see Fig. 1 of [8]).

Let us define $\Omega_{0}$ as the angular velocity about the Galactic center, assuming the Sun to follow a circular orbit (since the motion of the Sun around the Galaxy is clockwise in both our frames, $\Omega_{0}$ is negative, i.e., the vector is directed along $-\hat{z}$ ). If $\phi_{r}$ is an angle in the Galactic plane measured in the rotating frame from $\hat{x}^{\prime}$, and $\phi$ the corresponding angle measured in the fixed frame from $\hat{x}$ at time $t$, we have the relation: $\phi=\phi_{r}+\Omega_{0} t$ (see Fig. 1 of [8]). All the final results will be presented in the fixed frame.

The force $\mathbf{F}$ per unit of mass acting on a test particle orbiting the Sun under the influence of the Galactic tide is given by (see [10]):

$$
\begin{aligned}
\mathbf{F}= & -\frac{\mu M_{\odot}}{r^{3}} \mathbf{r}+(A-B)(3 A+B) x^{\prime} \hat{x}^{\prime}-(A-B)^{2} y^{\prime} \hat{y}^{\prime} \\
& -\left[4 \pi \mu \rho_{\odot}-2\left(B^{2}-A^{2}\right)\right] z \hat{z},
\end{aligned}
$$

where $x^{\prime}, y^{\prime}, z$ are the coordinates of the comet in the rotating frame, $\mathbf{r}$ is the Sun-comet vector of length $r, A$ and $B$ are the Oort constants, and $\rho_{\odot}$ is the local density of the Galactic disk in the solar neighbourhood. In the remaining of the paper we will assume $\rho_{0}=0.1 M_{\odot} \mathrm{pc}^{-3}[12]$ and an angular velocity of the Sun around the Galactic Center $\Omega_{0}=B-A=-26 \mathrm{kms}^{-1} \mathrm{kpc}^{-1}$, with the approximation $A=-B$.

The unit of mass is the solar mass $\left(M_{\odot}=1\right)$, the unit of time is the year, and the unit of length is the Astronomical Unit (AU). As a consequence, the gravitational constant $\mu$ is equal to $4 \pi^{2}$.

Let $\mathcal{G}_{1}, \mathcal{G}_{2}$ and $\mathcal{G}_{3}$ be defined by

$$
\begin{aligned}
& \mathcal{G}_{1}=-(A-B)(3 A+B) \\
& \mathcal{G}_{2}=(A-B)^{2} \\
& \mathcal{G}_{3}=4 \pi \mu \rho_{\odot}-2\left(B^{2}-A^{2}\right) .
\end{aligned}
$$


Then, with the chosen values of $A, B$ and $\rho_{0}$, one has:

$$
\begin{aligned}
& \mathcal{G}_{2}=-\mathcal{G}_{1}=7.0706 \times 10^{-16} \mathrm{yr}^{-2}, \\
& \mathcal{G}_{3}=5.6530 \times 10^{-15} \mathrm{yr}^{-2}, \\
& \Omega_{0}=-\sqrt{\mathcal{G}_{2}} .
\end{aligned}
$$

One may note that the relation $\mathcal{G}_{2}=-\mathcal{G}_{1}$ is a particular case which corresponds to a flat rotation curve of the Galaxy, i.e., to a constant tangential velocity of the stars around the galactic center whatever the star distance to the galactic center. Numerical experiments have shown that another choice of Oort constants $A$ and $B$ consistent with the observations does not affect the long term dynamics of the Oort cloud comets [9]. Consequently, some of the models presented here will use the relation $\mathcal{G}_{2}=-\mathcal{G}_{1}$ in order to simplify the equations.

The general equations of motion in Cartesian coordinates are:

$$
\begin{aligned}
& \frac{\mathrm{d}^{2} x}{\mathrm{~d} t^{2}}=-\frac{\mu M_{\odot}}{r^{3}} x-\mathcal{G}_{1} x^{\prime} \cos \left(\Omega_{0} t\right)+\mathcal{G}_{2} y^{\prime} \sin \left(\Omega_{0} t\right) \\
& \frac{\mathrm{d}^{2} y}{\mathrm{~d} t^{2}}=-\frac{\mu M_{\odot}}{r^{3}} y-\mathcal{G}_{1} x^{\prime} \sin \left(\Omega_{0} t\right)-\mathcal{G}_{2} y^{\prime} \cos \left(\Omega_{0} t\right) \\
& \frac{\mathrm{d}^{2} z}{\mathrm{~d} t^{2}}=-\frac{\mu M_{\odot}}{r^{3}} z-\mathcal{G}_{3} z,
\end{aligned}
$$

where $x, y, z$ are the coordinates of the comet in the fixed frame [thus $x^{\prime}=$ $x \cos \left(\Omega_{0} t\right)+y \sin \left(\Omega_{0} t\right)$ and $\left.y^{\prime}=-x \sin \left(\Omega_{0} t\right)+y \cos \left(\Omega_{0} t\right)\right]$. Equations (4) will be referred to as the Cartesian model, also denoted RADAU. The RADAU integrator described by $[7]$ is used at the 15 th order, with $L L=12$, to integrate Eqs. (4). This integrator was chosen because it is fast, reliable and accurate compared to other non-symplectic integrators.

\subsection{Regularised symplectic integrator}

This method was first introduced in [5]. For a detailed decription of the method one should read the original paper, only the key points are presented here. In the following we recall the key steps.

\section{Hamiltonian in Cartesian variables}

From Equation (1) the complete Hamiltonian is given by:

$$
\begin{aligned}
\mathcal{H} & =\mathcal{H}_{0}+\mathcal{H}_{1} \\
\mathcal{H}_{0} & =\frac{1}{2}\left(X^{2}+Y^{2}+Z^{2}\right)-\frac{\mu}{r}, \\
\mathcal{H}_{1} & =\frac{1}{2}\left(\mathcal{G}_{1} x^{\prime 2}+\mathcal{G}_{2} y^{2}+\mathcal{G}_{3} z^{2}\right),
\end{aligned}
$$

where $(X, Y, Z)^{T}$ is the velocity vector of the comet in the fixed frame. 
Introducing the first equation of system (3) explicitly, we can rewrite $\mathcal{H}_{1}$ as

$$
\mathcal{H}_{1}(x, y, z, t)=\frac{1}{2} \mathcal{G}_{2}\left[\left(y^{2}-x^{2}\right) C-2 x y S\right]+\frac{1}{2} \mathcal{G}_{3} z^{2},
$$

where

$$
C=\cos \left(2 \Omega_{0} t\right), \quad S=\sin \left(2 \Omega_{0} t\right) .
$$

It is well known that in cometary problems one cannot expect to meet moderate eccentricities of orbits; some kind of regularisation will become unavoidable if a fixed step integrator is to be applied. One of the standard regularising tools is the application of the so-called Kuustanheimo-Stiefel (KS) transformation that turns a Kepler problem into a harmonic oscillator at the expense of increasing the number of degrees of freedom [16]. The approach of [6] is used to set the KS variables in the canonical formalism.

\section{KS variables}

Leaving aside the in-depth quaternion interpretation of the KS transformation given in [6], we restrict ourselves to the basic set of transformation formulae, treating the KS variables as a formal column vector. In the phase space of the KS coordinates $\boldsymbol{u}=\left(u_{0}, u_{1}, u_{2}, u_{3}\right)^{\mathrm{T}}$ and KS momenta $\boldsymbol{U}=\left(U_{0}, U_{1}, U_{2}, U_{3}\right)^{\mathrm{T}}$, the former are defined by means of the inverse transformation

$$
\begin{aligned}
& x=\left(u_{0}^{2}+u_{1}^{2}-u_{2}^{2}-u_{3}^{2}\right) / \alpha, \\
& y=2\left(u_{1} u_{2}+u_{0} u_{3}\right) / \alpha, \\
& z=2\left(u_{1} u_{3}-u_{0} u_{2}\right) / \alpha,
\end{aligned}
$$

where $\alpha$ is an arbitrary parameter with the dimension of a length. A dimension raising transformation cannot be bijective, so the inverse of (10) is to some extent arbitrary. Following [6] we adopt

$$
\boldsymbol{u}=\sqrt{\frac{\alpha}{2(r+x)}}(0, r+x, y, z)^{\mathrm{T}},
$$

for $x \geq 0$, and

$$
\boldsymbol{u}=\sqrt{\frac{\alpha}{2(r-x)}}(-z, y, r-x, 0)^{\mathrm{T}},
$$

otherwise. A remarkable property of this transformation is that the distance $r$ becomes a quadratic function of $u_{i}$, namely

$$
r=\sqrt{x^{2}+y^{2}+z^{2}}=\frac{u_{0}^{2}+u_{1}^{2}+u_{2}^{2}+u_{3}^{2}}{\alpha}=\frac{\boldsymbol{u}^{2}}{\alpha} .
$$

The momenta conjugate to $\boldsymbol{u}$ are defined as

$$
\boldsymbol{U}=\frac{2}{\alpha}\left(\begin{array}{c}
u_{0} X+u_{3} Y-u_{2} Z \\
u_{1} X+u_{2} Y+u_{3} Z \\
-u_{2} X+u_{1} Y-u_{0} Z \\
-u_{3} X+u_{0} Y+u_{1} Z
\end{array}\right)
$$


The inverse transformation, allowing the computation of $\boldsymbol{R}=(X, Y, Z)^{\mathrm{T}}$,

$$
\boldsymbol{R}=\frac{1}{2 r}\left(\begin{array}{c}
u_{0} U_{0}+u_{1} U_{1}-u_{2} U_{2}-u_{3} U_{3} \\
u_{3} U_{0}+u_{2} U_{1}+u_{1} U_{2}+u_{0} U_{3} \\
-u_{2} U_{0}+u_{3} U_{1}-u_{0} U_{2}+u_{1} U_{3}
\end{array}\right),
$$

can be supplemented with the identity

$$
u_{1} U_{0}-u_{0} U_{1}-u_{3} U_{2}+u_{2} U_{3}=0 .
$$

In order to achieve the regularisation without leaving the canonical formalism, we have to change the independent variable from $t$ to a fictitious time $s$ and consider the extended phase space of dimension 10, with a new pair of conjugate variables $\left(u^{*}, U^{*}\right)$. Thus, in the extended set of canonical KS variables, the motion of a comet is governed by the Hamiltonian function

$$
\mathcal{M}=\frac{4 \boldsymbol{u}^{2}}{\alpha^{2}}\left(\mathcal{K}_{0}+U^{*}+\mathcal{K}_{1}\right)=0,
$$

where $\mathcal{K}_{0}$ and $\mathcal{K}_{1}$ stand for $\mathcal{H}_{0}$ and $\mathcal{H}_{1}$ expressed in terms of the extended $\mathrm{KS}$ variables set. The transformation just presented is univalent, hence the respective Hamiltonians will have different functional forms, but equal values: $\mathcal{H}_{0}=\mathcal{K}_{0}, \mathcal{H}_{1}=\mathcal{K}_{1}$. Restricting the motion to the manifold of $\mathcal{M}=0$ is of fundamental importance to the canonical change of independent variable; in practical terms we achieve it by setting

$$
U^{*}=-\mathcal{K}_{0}-\mathcal{K}_{1}
$$

at the beginning of the numerical integration.

Splitting the Hamiltonian function $\mathcal{M}$ into the sum of the principal term $\mathcal{M}_{0}$ and of a perturbation $\mathcal{M}_{1}$, we have

$$
\begin{aligned}
& \mathcal{M}_{0}=\frac{1}{2} \boldsymbol{U}^{2}+\left(4 U^{*} / \alpha^{2}\right) \boldsymbol{u}^{2}, \\
& \mathcal{M}_{1}=\frac{4 \boldsymbol{u}^{2}}{\alpha^{2}} \mathcal{H}_{1}(x, y, z, t) .
\end{aligned}
$$

Although nothing prohibits $u^{*}$ and $t$ from differing by an additive constant, we do not take advantage from this freedom and will therefore use the symbol $t$ in most instances instead of the formal $u^{*}$. In the next Section we provide equations of motion generated by $\mathcal{M}_{0}$ and $\mathcal{M}_{1}$ alone; the complete equations of motion can be quickly obtained by adding the respective right-hand sides.

\section{Keplerian motion}

The principal virtue of the KS variables consists in their ability to transform the Kepler problem into a four-dimensional oscillator with a constant frequency 


$$
\omega=2 \frac{\sqrt{2 U^{*}}}{\alpha}
$$

In addition one gets the equation for the fictitious time $s$, that may be written as:

$$
\frac{\mathrm{d} s}{\mathrm{~d} t}=\frac{\alpha}{4 r}
$$

Thanks to the introduction of $\alpha$, the fictitious time $s$ has the dimension of time and if we assume

$$
\alpha=\frac{2 \mu}{\left|U^{*}\right|},
$$

orbital periods in $s$ and $t$ will be equal.

For $U^{*}>0$, the map $\Phi_{0}$ representing the solution of the Keplerian motion can be directly quoted from [2]. If $\Delta$ is the fictitious time step, then

$$
\Phi_{0, \Delta}:\left(\begin{array}{c}
\boldsymbol{u} \\
\boldsymbol{U} \\
U^{*}
\end{array}\right) \rightarrow\left(\begin{array}{c}
\boldsymbol{u} \cos \omega \Delta+\boldsymbol{U} \omega^{-1} \sin \omega \Delta \\
-\boldsymbol{u} \omega \sin \omega \Delta+\boldsymbol{U} \cos \omega \Delta \\
U^{*}
\end{array}\right)
$$

Moreover, if $\boldsymbol{v}=\Phi_{0, \Delta} \boldsymbol{u}$ and $\boldsymbol{V}=\Phi_{0, \Delta} \boldsymbol{U}$ are the final values of variables,

$$
\Phi_{0, \Delta}: t \rightarrow t+\frac{2 \Delta}{\alpha^{2}}\left(\boldsymbol{u}^{2}+\frac{\boldsymbol{U}^{2}}{\omega^{2}}\right)+2 \frac{\boldsymbol{u}^{\mathrm{T}} \boldsymbol{U}-\boldsymbol{v}^{\mathrm{T}} \boldsymbol{V}}{\alpha^{2} \omega^{2}} .
$$

One may easily check that the sum $\boldsymbol{u}^{2}+\boldsymbol{U}^{2} \omega^{-2}$ is invariant under $\Phi_{0}$ and it can be replaced by $\boldsymbol{v}^{2}+\boldsymbol{V}^{2} \omega^{-2}$ in practical computations of the Kepler equation (25).

It may happen, however, that $U^{*}<0$ (when the motion is hyperbolic for instance). A simple modification of $\Phi_{0}$ in that case amounts to take

$$
\omega=2 \frac{\sqrt{-2 U^{*}}}{\alpha},
$$

and replacing equations (24) and (25) by

$$
\Phi_{0, \Delta}:\left(\begin{array}{c}
\boldsymbol{u} \\
\boldsymbol{U} \\
U^{*}
\end{array}\right) \rightarrow\left(\begin{array}{c}
\boldsymbol{u} \cosh \omega \Delta+\boldsymbol{U} \omega^{-1} \sinh \omega \Delta \\
\boldsymbol{u} \omega \sinh \omega \Delta+\boldsymbol{U} \cosh \omega \Delta \\
U^{*}
\end{array}\right)
$$

and

$$
\Phi_{0, \Delta}: t \rightarrow t+\frac{2 \Delta}{\alpha^{2}}\left(\boldsymbol{u}^{2}-\frac{\boldsymbol{U}^{2}}{\omega^{2}}\right)-2 \frac{\boldsymbol{u}^{\mathrm{T}} \boldsymbol{U}-\boldsymbol{v}^{\mathrm{T}} \boldsymbol{V}}{\alpha^{2} \omega^{2}} .
$$

Similarly to the elliptic case, $\boldsymbol{u}^{2}-\boldsymbol{U}^{2} / \omega^{2}$ is invariant under $\Phi_{0}$. 


\section{Galactic tide}

The Hamiltonian $\mathcal{M}_{1}$ has the nice property of being independent from the momenta. Thus a half of the equations of motion have right-hand sides equal to zero, and the remaining right-hand sides are constant.

Accordingly, all KS coordinates are constant, the physical time $t$ does not flow, and the momenta are subjected to a linear 'kick':

$$
\Phi_{1, \Delta}:\left(\begin{array}{c}
\boldsymbol{u} \\
t \\
\boldsymbol{U} \\
U^{*}
\end{array}\right) \rightarrow\left(\begin{array}{c}
\boldsymbol{u} \\
t \\
\boldsymbol{U}-\Delta \boldsymbol{F}(\boldsymbol{u}, t) \\
U^{*}-\Delta F^{*}(\boldsymbol{u}, t)
\end{array}\right)
$$

Mixing Cartesian and KS variables for the sake of brevity, we can represent $\boldsymbol{F}$ and $F^{*}$ as

$$
\begin{aligned}
\boldsymbol{F} & =\frac{8 \mathcal{H}_{1}}{\alpha^{2}} \boldsymbol{u}+\frac{4 \boldsymbol{u}^{2}}{\alpha^{2}} \frac{\partial \mathcal{H}_{1}}{\partial \boldsymbol{u}} \\
F^{*} & =\frac{4 \boldsymbol{u}^{2}}{\alpha^{2}} \Omega_{0} \mathcal{G}_{2} \xi_{3},
\end{aligned}
$$

where

$$
\begin{aligned}
\frac{\partial \mathcal{H}_{1}}{\partial \boldsymbol{u}} & =-\mathcal{G}_{2} \xi_{2} \frac{\partial x}{\partial \boldsymbol{u}}+\mathcal{G}_{2} \xi_{1} \frac{\partial y}{\partial \boldsymbol{u}}+\mathcal{G}_{3} z \frac{\partial z}{\partial \boldsymbol{u}} \\
\xi_{1} & =y C-x S, \\
\xi_{2} & =x C+y S \\
\xi_{3} & =\left(x^{2}-y^{2}\right) S-2 x y C .
\end{aligned}
$$

\section{Symplectic corrector}

One of the advantages offered by the integrators introduced in [11] is a simple definition of a symplectic corrector - an extra stage that improves the accuracy in perturbed motion problems. The symplectic corrector is defined as a solution of the equations of motion generated by

$$
\mathcal{M}_{\mathrm{c}}=\left\{\left\{\mathcal{M}_{0}, \mathcal{M}_{1}\right\}, \mathcal{M}_{1}\right\} \text {, }
$$

where $\{$,$\} is the canonical (or 'symplectic') Poisson bracket in the phase space$ spanned by $\boldsymbol{u}, t, \boldsymbol{U}, U^{*}$. Observing that $\mathcal{M}_{0}$ is quadratic in $\boldsymbol{U}$ and linear in $U^{*}$, we easily obtain

$$
\mathcal{M}_{\mathrm{c}}(\boldsymbol{u}, t)=\sum_{i=0}^{3}\left(\frac{\partial \mathcal{M}_{1}}{\partial u_{i}}\right)^{2}=\boldsymbol{F}^{2} .
$$

The solution of the equations of motion derived from $\mathcal{M}_{\mathrm{c}}$ results in: 


$$
\Phi_{\mathrm{c}, \Delta}:\left(\begin{array}{c}
\boldsymbol{u} \\
t \\
U_{j} \\
U^{*}
\end{array}\right) \rightarrow\left(\begin{array}{c}
\boldsymbol{u} \\
t \\
U_{j}-2 \Delta\left(\frac{\partial \boldsymbol{F}}{\partial u_{j}}\right) \cdot \boldsymbol{F} \\
U^{*}-2 \Delta\left(\frac{\partial \boldsymbol{F}}{\partial t}\right) \cdot \boldsymbol{F}
\end{array}\right) .
$$

In spite of a formally simple form, equations (36) involve rather complicated expressions for the second derivatives of $\mathcal{M}_{1}$, because

$$
\begin{aligned}
& \frac{\partial \boldsymbol{F}}{\partial u_{j}} \cdot \boldsymbol{F}(\boldsymbol{u}, t)=\sum_{i=0}^{3} \frac{\partial^{2} \mathcal{M}_{1}}{\partial u_{i} \partial u_{j}} \frac{\partial \mathcal{M}_{1}}{\partial u_{i}} \\
& \frac{\partial \boldsymbol{F}}{\partial t} \cdot \boldsymbol{F}(\boldsymbol{u}, t)=\sum_{i=0}^{3} \frac{\partial^{2} \mathcal{M}_{1}}{\partial u_{i} \partial t} \frac{\partial \mathcal{M}_{1}}{\partial u_{i}} .
\end{aligned}
$$

One may find a detailed method to compute the Hessian matrix of $\mathcal{M}_{1}$ in [5].

\section{Laskar-Robutel integrators}

The composition methods of [11] differ from usual recipes because, regardless of the number of 'stages' involved in one step, they all remain second-order integrators according to the formal estimates. However, if the Hamiltonian has been split into a leading term and a perturbation having a small parameter $\varepsilon$ as a factor, the truncation error of the integrator is $\max \left(\varepsilon^{2} h^{3}, \varepsilon h^{m}\right)$ where $m$ is the number of stages involved in one step. The second term of this sum is similar to classical composition methods errors, and the first can be quite small for weakly perturbed problems. At the expense of the $\varepsilon^{2} h^{3}$ term in the error estimate, the authors were able to avoid backward stages that degrade numerical properties of usual composition methods. The use of a corrector improves the integrator by reducing the truncation error: its first term drops to $\varepsilon^{2} h^{5}$.

Following the recommendation of [11], and after having performed numerical tests (see [5]), the best integrator is obtained using the following composition for each single step of size $h$ :

$$
\begin{aligned}
\Phi_{h}= & \Phi_{\mathrm{c}, q} \circ \Phi_{1, d_{1}} \circ \Phi_{0, c_{2}} \circ \Phi_{1, d_{2}} \circ \Phi_{0, c_{3}} \circ \\
& \circ \Phi_{1, d_{2}} \circ \Phi_{0, c_{2}} \circ \Phi_{1, d_{1}} \circ \Phi_{\mathrm{c}, q},
\end{aligned}
$$

where

$$
\begin{array}{ll}
d_{1}=h / 12, & d_{2}=(5 / 12) h, \\
c_{2}=(1 / 2-\sqrt{5} / 10) h, & c_{3}=h / \sqrt{5}, \\
q=-h^{3}(3861-791 \sqrt{21}) / 576 . &
\end{array}
$$

This integrator will be referred as LARKS. 


\section{LARKS step size choice}

The Hamiltonian error of LARKS, based on the above composition, is proportional to $\varepsilon^{2} h^{4}$ (see [5]). Observing that $\varepsilon \propto a r^{2}$, where $a$ is the semi-major axis of a comet, and $r$ is the Sun-comet distance, we look for the step size selection rule that gives a similar precision for a wide range of initial conditions. This can be achieved if the product

$$
K=\varepsilon^{2} h^{4},
$$

has similar values for all comets to be studied. Thus, finding some optimum step size $h_{\mathrm{o}}$ for a given semi-axis $a_{\mathrm{o}}$, and then launching the integration for a different semi-axis $a_{1}$, we adjust the step size and use

$$
h_{1}=h_{\mathrm{o}}\left(\frac{a_{\mathrm{o}}}{a_{1}}\right)^{3 / 2},
$$

when the orbit is elliptic $\left(a_{1}>0\right)$ and using $a_{1}$ instead of $r$. For a hyperbolic orbit the step size is adjusted through:

$$
h_{1}=h_{\mathrm{o}}\left(\frac{a_{\mathrm{o}}^{3 / 2}}{\left|a_{1}\right|^{1 / 2} r}\right) .
$$

In the test described in this Section, we set $h_{\mathrm{o}}$ as $1 / 20$ of the Keplerian period implied by $a_{\mathrm{o}}=50000 \mathrm{AU}$ and adjusted the step according to (42) or (43) for other orbits. For elliptical orbits, in order to avoid numerical resonance between the step size and the orbital period [19], we do not use a step size larger than $1 / 20$ of the Keplerian period, even if it might be allowed by Eq. (42).

\section{Stop time for LARKS}

The fact that the fictitious time $s$ is the independent variable is an inevitable issue associated with the use of the KS variables regularisation. What happens if one wants to obtain the state of a comet at some particular final epoch of the physical time $t$ ? This problem appears if one wants to stop the integration as close as possible to a precise value $T_{f}$ of the real time.

A method which turns out to be effective, whatever the dynamics, is the following. Let $f$ be the function defined by $f(s)=t-T_{f}$. Thus the problem is to solve $f(s)=0$. Let $f_{p}$ and $s_{p}$ be the values of $f$ and $s$ before some step, and $f_{a}$ and $s_{a}$ be the values after this step. The integration stops as soon as $f_{a}>0$. From this point, the method is built according to an iterative process which evaluates $f_{p}, s_{p}$ and $f_{a}, s_{a}$, such that the solution $s_{s}$ is always between $s_{p}$ and $s_{a}$.

For each step, one computes the derivatives $d f_{p}$ and $d f_{a}$ of $f$ in $s_{p}$ and $s_{a}$ respectively, using Eqs. (22) and (13). Consequently, one can easily compute the equation of the tangent to $f$ in $s_{p}$ and $s_{a}$. Let $s_{m}$ be the value of $s$ for 
which the two tangents intersects. If $s_{m}$ is not between $s_{p}$ and $s_{a}$, the next guess $s_{g}$ of the solution $s_{s}$ is computed using a linear approximation of $f$ or a bisection method between $s_{p}$ and $s_{a}$. The choice is made according to the method which makes the most important reduction of the interval $\left[s_{p}, s_{a}\right]$. Otherwise, if $s_{m}$ is between $s_{p}$ and $s_{a}$, then the next guess $s_{g}$ is given by the intersection of the tangent at $f$ in $s_{p}$ (resp. in $s_{a}$ ) with the abscissa axis if it lies between $s_{p}$ and $s_{m}$ (resp. between $s_{m}$ and $s_{a}$ ).

One stops the iterative process as soon as $f\left(s_{g}\right)$ is close enough to 0 , that is $t_{g}-T_{f} \approx 0$, where $t_{g}$ is the value of the real time obtained for $s_{g}$.

\section{Stop at perihelion for LARKS}

In the framework of Oort cloud comets dynamics, it may be necessary to suspend the integration of a comet at its perihelion. When an integrator like RADAU is used, then the step size is very small when the comet passes through its perihelion, thus it is quite easy to stop at the cometary perihelion only by checking the evolution of the Sun-comet distance. This is not the case for LARKS, which may have a large step size even when the comets is at its perihelion, hence the evolution of the Sun-comet distance is not sufficient.

However, when a comet is near its perihelion, one may neglect the perturbative part due to the galactic tide. Consequently, as it has been already noted, the motion in the KS variables is simply a harmonic oscillator. Using this property it is very easy to stop the integration exactly at the cometary perihelion. Indeed, if $(\boldsymbol{u}, \boldsymbol{U})$ are the KS variables of a comet, then $\boldsymbol{u} \cdot \boldsymbol{U}=0$ when the comet is at its perihelion. Thus, let $\left(\boldsymbol{u}_{p}, \boldsymbol{U}_{p}\right)$ be the KS variables before some step, and $\left(\boldsymbol{u}_{a}, \boldsymbol{U}_{a}\right)$ after this step. When $\boldsymbol{u}_{p} \cdot \boldsymbol{U}_{p}<0$ and $\boldsymbol{u}_{a} \cdot \boldsymbol{U}_{a}>0$ it means that the comet went through its perihelion during the step. When $U^{*}>0$, using Eq. 24, the step length $h$ which should be performed from $\left(\boldsymbol{u}_{p}, \boldsymbol{U}_{p}\right)$ to the exact perihelion is given by:

$$
h=\frac{1}{2 \omega} \tan ^{-1}\left(\frac{2 \omega \boldsymbol{u}_{p} \cdot \boldsymbol{U}_{p}}{\omega^{2} u_{p}^{2}-U_{p}^{2}}\right) .
$$

When $U^{*}<0$, using Eq. 25, the step length is given by:

$$
h=\frac{1}{4 \omega} \log \left(\frac{1+X}{1-X}\right),
$$

where

$$
X=\frac{2 \omega \boldsymbol{u}_{p} \cdot \boldsymbol{U}_{p}}{\omega^{2} u_{p}^{2}+U_{p}^{2}} .
$$

\subsection{The Hamiltonian models}

In this section we will give the Hamiltonian equations of motion derived from the average Hamiltonian, using two sets of variables according to the value of the orbital eccentricity $e$ (see [9]). 


\section{The Hamiltonian model with Delaunay variables}

The complete Hamiltonian given by:

$$
\mathcal{H}=-\frac{\mu}{2 a}+\mathcal{G}_{1} \frac{x^{\prime 2}}{2}+\mathcal{G}_{2} \frac{y^{\prime 2}}{2}+\mathcal{G}_{3} \frac{z^{2}}{2},
$$

may be written using the Delaunay's variables: $L=\sqrt{\mu a}, G=\sqrt{\mu a\left(1-e^{2}\right)}$, $H=G \cos i, \ell=M, g=\omega$ and $h=\Omega$, where $a, e, i, M, \omega, \Omega$ are the cometary semi-major axis, eccentricity, inclination, mean anomaly, argument of perihelion and longitude of node (all the angles being measured in the fixed Galactic frame). The mean anomaly being a fast variable with respect to the other ones, the Hamiltonian is averaged over $\ell$.

Then, one writes the averaged Hamiltonian equations of motion, which gives:

$$
\begin{aligned}
&\left\langle\frac{\mathrm{d} L}{\mathrm{~d} t}\right\rangle= 0 \\
&\left\langle\frac{\mathrm{d} G}{\mathrm{~d} t}\right\rangle=-\frac{5 L^{2}}{2 \mu^{2}}\left(L^{2}-G^{2}\right)\left\{\operatorname { c o s } g \operatorname { s i n } g \left[\mathcal{G}_{3}\left(1-\frac{H^{2}}{G^{2}}\right)\right.\right. \\
&+\left(\mathcal{G}_{1} \sin ^{2} h_{r}+\mathcal{G}_{2} \cos ^{2} h_{r}\right) \frac{H^{2}}{G^{2}}-\mathcal{G}_{1} \cos ^{2} h_{r} \\
&\left.\left.-\mathcal{G}_{2} \sin ^{2} h_{r}\right]-\left(\mathcal{G}_{1}-\mathcal{G}_{2}\right)\left(\cos ^{2} g-\sin ^{2} g\right) \cos h_{r} \sin h_{r} \frac{H}{G}\right\} \\
&\left\langle\frac{\mathrm{d} H}{\mathrm{~d} t}\right\rangle= \frac{L^{2}}{2 \mu^{2}}\left(\mathcal{G}_{1}-\mathcal{G}_{2}\right)\left\{5\left(L^{2}-G^{2}\right) \frac{H}{G} \cos g \sin g\left(\cos ^{2} h_{r}-\sin ^{2} h_{r}\right)\right. \\
&+\sin h_{r} \cos h_{r} \\
&\left.\cdot\left[G^{2}-H^{2}+5\left(L^{2}-G^{2}\right)\left(\cos ^{2} g-\sin ^{2} g \frac{H^{2}}{G^{2}}\right)\right]\right\} \\
&\left\langle\frac{\mathrm{d} g}{\mathrm{~d} t}\right\rangle= \frac{L^{2} G}{2 \mu^{2}}\left\{\mathcal{G}_{3}\left[1-5 \sin ^{2} g\left(1-\frac{L^{2} H^{2}}{G^{4}}\right)\right]\right. \\
&+\left(\mathcal{G}_{1} \cos ^{2} h_{r}+\mathcal{G}_{2} \sin ^{2} h_{r}\right)\left(1-5 \cos ^{2} g\right) \\
&-5\left(\mathcal{G}_{1} \sin ^{2} h_{r}+\mathcal{G}_{2} \cos ^{2} h_{r}\right) \frac{L^{2} H^{2}}{G^{4}} \sin ^{2} g \\
&\left.+5\left(\mathcal{G}_{1}-\mathcal{G}_{2}\right) \cos g \sin ^{2} \cos h_{r} \sin _{r}\left(G^{2}+L^{2}\right) \frac{H}{G^{3}}\right\} \\
& \frac{L^{2}}{2 G \mu^{2}}\left\{\left(\mathcal{G}_{1} \sin ^{2} h_{r}+\mathcal{G}_{2} \cos { }^{2} h_{r}-\mathcal{G}_{3}\right)\right. \\
&\left\langle\frac{\mathrm{d} h}{\mathrm{~d} t}\right\rangle {\left[G^{2}+5\left(L^{2}-G^{2}\right) \sin ^{2} g\right] \frac{H}{G}-5\left(\mathcal{G}_{1}-\mathcal{G}_{2}\right) } \\
&\left.\left(L^{2}-G^{2}\right) \cos g \sin ^{2} \cos h_{r} \sin _{r}\right\} \\
& \hline
\end{aligned}
$$


where $h_{r}=h-\Omega_{0} t$ at time $t$.

The quantity $L$, i.e. the semi-major axis, is obviously conserved since the mean anomaly does not appear in the averaged Hamiltonian. Furthermore, one may note that, when the radial component of the tide is neglected, i.e. when $\mathcal{G}_{1}=\mathcal{G}_{2}=0$, then $H$ is conserved. In this case the dynamics is completely integrable. Many papers were devoted to this peculiar case: for instance [3, $10,14,15]$ and [1].

\section{The Hamiltonian model with Matese elements}

When $e \simeq 1$, Equations (49-53) become singular. In order to remove this singularity, we adopt the variables: $L, \Theta=H_{b}, H, M, \theta=b$ and $\lambda=l$, with: $H_{b}=-\sqrt{\mu a\left(1-e^{2}\right)} \cos \alpha$ and $H=\sqrt{\mu a\left(1-e^{2}\right)} \cos i$. Here $b$ and $l$ are the latitude and longitude of perihelion of the comet, and $\alpha$ is the angle between the orbital plane and the plane orthogonal to the Galactic plane and passing through the perihelion and the Galactic poles, measured from the south Galactic pole to the cometary velocity (see Fig. 1 of [9]).

This set of elements will be referred to as Matese elements since it first appeared in [15]. Similar elements have been used elsewhere in the literature in order to remove the singularity at $e=1$ : see [18] for elliptic collision orbits, and [17] for hyperbolic collision orbits. The Matese elements are slightly different from those used in the quoted papers, but the procedure to define them is similar. The can be shown to be canonical.

One substitutes $x^{\prime}, y^{\prime}$ and $z$ by the Matese elements in Eq. (47), and averages with respect to the mean anomaly. Then, the Hamiltonian equations of motion are:

$$
\begin{aligned}
\left\langle\frac{\mathrm{d} L}{\mathrm{~d} t}\right\rangle= & 0 \\
\left\langle\frac{\mathrm{d} \Theta}{\mathrm{d} t}\right\rangle= & \frac{L^{2}}{2 \mu^{2}}\left[\cos \theta \sin \theta\left(-4 \Theta^{2}+5 L^{2}\right)\left(\mathcal{G}_{1} \cos ^{2} \lambda_{r}+\mathcal{G}_{2} \sin ^{2} \lambda_{r}\right)\right. \\
& -\mathcal{G}_{1}\left(\Theta \cos \theta \cos \lambda_{r}+H \sin \lambda_{r} \frac{\sin \theta}{\cos ^{2} \theta}\right) \\
& \cdot\left(\Theta \sin \theta \cos \lambda_{r}+H \sin \lambda_{r} \frac{1}{\cos \theta}\right) \\
& -\mathcal{G}_{2}\left(\Theta \cos \theta \sin \lambda_{r}-H \cos \lambda_{r} \frac{\sin \theta}{\cos ^{2} \theta}\right) \\
& \cdot\left(\Theta \sin \theta \sin \lambda_{r}-H \cos \lambda_{r} \frac{1}{\cos \theta}\right) \\
& \left.+\mathcal{G}_{3} \frac{\sin \theta}{\cos ^{3} b}\left(5\left(\Theta^{2}-L^{2}\right) \cos ^{4} \theta+4 H^{2}\right)\right] \\
\left\langle\frac{\mathrm{d} H}{\mathrm{~d} t}\right\rangle= & \frac{L^{2}\left(\mathcal{G}_{1}-\mathcal{G}_{2}\right)}{2 \mu^{2} \cos ^{2} \theta}\left[\left(-4 \Theta^{2} \cos ^{2} \theta-4 H^{2}+5 L^{2} \cos ^{2} \theta\right) \cos ^{2} \theta \cos \lambda_{r} \sin \lambda_{r}\right.
\end{aligned}
$$




$$
\begin{aligned}
& \left.+\left(\Theta \cos \theta \sin \theta \sin \lambda_{r}-H \cos \lambda_{r}\right)\left(\Theta \cos \theta \sin \theta \cos \lambda_{r}+H \sin \lambda_{r}\right)\right] \\
\left\langle\frac{\mathrm{d} \theta}{\mathrm{d} t}\right\rangle= & \frac{L^{2}}{2 \mu^{2} \cos ^{2} \theta}\left\{-4 \Theta \cos ^{4} \theta\left(\mathcal{G}_{1} \cos ^{2} \lambda_{r}+\mathcal{G}_{2} \sin ^{2} \lambda_{r}\right)\right. \\
& +\mathcal{G}_{1}\left(\Theta \cos \theta \sin \theta \cos \lambda_{r}+H \sin \lambda_{r}\right) \cos \theta \sin \theta \cos \lambda_{r} \\
& +\mathcal{G}_{2}\left(\Theta \cos \theta \sin \theta \sin \lambda_{r}-H \cos \lambda_{r}\right) \cos \theta \sin \theta \sin \lambda_{r} \\
& \left.+\mathcal{G}_{3} \Theta \cos ^{2} \theta\left(1-5 \sin ^{2} \theta\right)\right\} \\
\left\langle\frac{\mathrm{d} \lambda}{\mathrm{d} t}\right\rangle= & \frac{L^{2}}{2 \mu^{2} \cos ^{2} \theta}\left\{-4 H \cos ^{2} \theta\left(\mathcal{G}_{1} \cos ^{2} \lambda_{r}+\mathcal{G}_{2} \sin ^{2} \lambda_{r}\right)\right. \\
& +\mathcal{G}_{1} \sin \lambda_{r}\left(\Theta \cos \theta \sin \theta \cos \lambda_{r}+H \sin \lambda_{r}\right) \\
& \left.-\mathcal{G}_{2} \cos \lambda_{r}\left(\Theta \cos \theta \sin \theta \sin \lambda_{r}-H \cos \lambda_{r}\right)-4 \mathcal{G}_{3} H \sin ^{2} \theta\right\}
\end{aligned}
$$

The singularity at $\cos \theta=0$ is evident, but these equations show that the singularity at $e=1$ has indeed disappeared.

\section{The Mappings}

The two above averaged models are already faster than the Cartesian model (see [8]). However, in order to enhance their efficiency, on may consider the truncated Taylor development of their solution. More precisely, one writes any of the averaged models in the form:

$$
\frac{\mathrm{d} \mathbf{x}}{\mathrm{d} t}=\mathbf{f}\left(\mathbf{x}_{r}\right),
$$

where $\mathbf{x}$ is the vector defined by the Delaunay or the Matese elements, and the subscript $r$ means that the longitude is measured in the rotating frame. Then, the truncated Taylor development at order $N$ of the solution of this equation is:

$$
\mathbf{x}(T+\Delta T)=\mathbf{x}(T)+\left.\sum_{n=1}^{N} \frac{\mathrm{d}^{n} \mathbf{x}}{\mathrm{d} t^{n}}\right|_{T} \frac{\Delta T^{n}}{n !}+\mathcal{O}\left(\Delta T^{N+1}\right),
$$

where the subscript $T$ indicates that the quantities are computed at time $T$.

Taking $\Delta T$ equal to one orbital period of the comet (which is consistent with having averaged the Hamiltonian) this development provides us an easy way to derive mappings of different orders.

Experiments have shown (see [8]) that the mappings of order 3 give the best compromise between precision and velocity. However, one should be very careful in using these mappings, due to the singularities of the two averaged models. Indeed, the effects of the singularity when $e=1$ for the model using the Delaunay variables and the singularity when $\cos b=0$ for the model using the Matese variables are enhanced when one uses the truncated Taylor development of their solution. 
It turns out that the mapping using the Delaunay elements may be safely used for eccentricity smaller than 0.999, otherwise the mapping using the Matese elements is more precise. The composition of this two mappings will be referred as the $M A P P$ model.

\subsection{The Lie-Poisson model}

This model is described in detail in [5]. One should refer to this paper for a full description of the method.

\section{Equations of motion}

The integrators presented in the previous Sections solve the equations of motion in the fixed reference frame, where the radial component of the Galactic tide is explicitly time-dependent. Our second method can be more conveniently discussed in the rotating heliocentric reference frame $\left(\hat{x}^{\prime}, \hat{y}^{\prime}, \hat{z}\right)$. The present model uses also the first equation of (3) explicitly, which simplifies drastically the results.

The Hamiltonian function for a comet subjected to the Galactic tide in the rotating frame is given by:

$$
\begin{aligned}
\mathcal{H} & =\mathcal{H}_{0}+\mathcal{H}_{1}, \\
\mathcal{H}_{0} & =\frac{1}{2}\left(X^{\prime 2}+Y^{\prime 2}+Z^{2}\right)-\frac{\mu}{\left(x^{\prime 2}+y^{\prime 2}+z^{2}\right)^{\frac{1}{2}}}, \\
\mathcal{H}_{1} & =\Omega_{0}\left(y^{\prime} X^{\prime}-x^{\prime} Y^{\prime}\right)+\frac{1}{2}\left(\mathcal{G}_{2}\left(y^{\prime 2}-x^{\prime 2}\right)+\mathcal{G}_{3} z^{2}\right),
\end{aligned}
$$

where $\left(x^{\prime}, y^{\prime}, z\right)$ and $\left(X^{\prime}, Y^{\prime}, Z\right)$ are the position and velocity of the comet in the rotating frame.

As in Section 2.3, one may average the Hamiltonian $\mathcal{H}$ with respect to the mean anomaly $\ell$. The averaged Hamiltonian $\left\langle\mathcal{H}_{1}\right\rangle$ is now expressed in terms of the Laplace vector $\boldsymbol{e}$ and a scaled angular momentum vector $\boldsymbol{h}$ instead of canonical elements as the Delaunay or the Matese ones. Their components are related to the Keplerian orbit elements

$$
\begin{gathered}
\boldsymbol{e} \equiv\left(\begin{array}{l}
e_{1} \\
e_{2} \\
e_{3}
\end{array}\right)=e\left(\begin{array}{c}
\cos \omega \cos \Omega_{r}-c \sin \omega \sin \Omega_{r} \\
\cos \omega \sin \Omega_{r}+c \sin \omega \cos \Omega_{r} \\
s \sin \omega
\end{array}\right), \\
\boldsymbol{h} \equiv\left(\begin{array}{l}
h_{1} \\
h_{2} \\
h_{3}
\end{array}\right)=\sqrt{1-e^{2}}\left(\begin{array}{c}
s \sin \Omega_{r} \\
-s \cos \Omega_{r} \\
c
\end{array}\right),
\end{gathered}
$$

where $e$ is the eccentricity, $s=\sin i, c=\cos i$. Recalling that in the rotating frame the momenta $X^{\prime}$ and $Y^{\prime}$ are not equal to velocities $\frac{\mathrm{d} x^{\prime}}{\mathrm{d} t}$ and $\frac{\mathrm{d} y^{\prime}}{\mathrm{d} t}$ (this fact that can be immediately deduced from the canonical equations $\frac{\mathrm{d} x^{\prime}}{\mathrm{d} t}=\partial \mathcal{H} / \partial X^{\prime}$ 
and $\left.\frac{\mathrm{d} y^{\prime}}{\mathrm{d} t}=\partial \mathcal{H} / \partial Y^{\prime}\right)$, we assume that the usual transformation rules between Keplerian elements and position/velocity are used with the velocities directly substituted by the momenta. With this approach the Keplerian motion in the rotating frame is described by means of orbital elements that are all constant except for $\Omega_{r}$ which reflects the frame rotation $\left(\frac{\mathrm{d} \Omega_{r}}{\mathrm{~d} t}=-\Omega_{0}\right)$.

Using the 'vectorial elements' $\boldsymbol{h}$ and $\boldsymbol{e}$, letting $n$ stand for

$$
n=\sqrt{\frac{\mu}{a^{3}}}
$$

and changing the independent variable from time $t$ to $\tau_{1}$, such that

$$
\frac{\mathrm{d} \tau_{1}}{\mathrm{~d} t}=\frac{\mathcal{G}_{3}}{n} .
$$

one obtains the averaged Hamiltonian $\langle\mathcal{H}\rangle$, given by

$$
\begin{aligned}
\langle\mathcal{H}\rangle= & n a^{2}\left[\frac{5}{4} e_{3}^{2}+\frac{1}{4} h_{1}^{2}+\frac{1}{4} h_{2}^{2}+\right. \\
& \left.+\nu\left(-\frac{5}{4} e_{1}^{2}+\frac{5}{4} e_{2}^{2}+\frac{1}{4} h_{1}^{2}-\frac{1}{4} h_{2}^{2}-n \Omega_{0}^{-1} h_{3}\right)\right],
\end{aligned}
$$

where, all the constant terms have been dropped and, using the usual approximation $\Omega_{0}=-\sqrt{\mathcal{G}_{2}}$, we introduced a dimensionless parameter

$$
\nu=\frac{\Omega_{0}^{2}}{\mathcal{G}_{3}}=\frac{\mathcal{G}_{2}}{\mathcal{G}_{3}} .
$$

The vectorial elements can be used to create a Lie-Poisson bracket

$$
(f ; g) \equiv\left(\frac{\partial f}{\partial \boldsymbol{v}}\right)^{\mathrm{T}} \mathbf{J}(\boldsymbol{v}) \frac{\partial g}{\partial \boldsymbol{v}}
$$

with the structure matrix

$$
\mathbf{J}(\boldsymbol{v})=\left(\begin{array}{c}
\hat{\mathbf{h}} \hat{\mathbf{e}} \\
\hat{\mathbf{e}} \hat{\mathbf{h}}
\end{array}\right) \text {. }
$$

The 'hat map' of any vector $\boldsymbol{x}=\left(x_{1}, x_{2}, x_{3}\right)^{\mathrm{T}}$ is defined as

$$
\hat{\mathbf{x}}=\left(\begin{array}{ccc}
0 & -x_{3} & x_{2} \\
x_{3} & 0 & -x_{1} \\
-x_{2} & x_{1} & 0
\end{array}\right)
$$

This matrix is known as the vector product matrix, because

$$
\hat{\mathrm{x}} \boldsymbol{y}=\boldsymbol{x} \times \boldsymbol{y} .
$$

Using the Lie-Poisson bracket (65) we can write equations of motion for the vectorial elements

$$
\boldsymbol{v}=\left(h_{1}, h_{2}, h_{3}, e_{1}, e_{2}, e_{3}\right)^{\mathrm{T}},
$$


in the non-canonical Hamiltonian form

$$
\boldsymbol{v}^{\prime}=(\boldsymbol{v} ; \mathcal{K})
$$

where derivatives with respect to $\tau$ are marked by the 'prime' symbol and the scaled Hamiltonian

$$
\mathcal{K}=-\frac{\langle\mathcal{H}\rangle}{n a^{2}} .
$$

Writing equations (70) explicitly, we obtain

$$
\begin{aligned}
h_{1}^{\prime} & =-\frac{5}{2}(1-\nu) e_{2} e_{3}+\frac{1-\nu}{2} h_{2} h_{3}+\frac{n \nu}{\Omega_{0}} h_{2}, \\
h_{2}^{\prime} & =\frac{5}{2}(1+\nu) e_{1} e_{3}-\frac{1+\nu}{2} h_{1} h_{3}-\frac{n \nu}{\Omega_{0}} h_{1}, \\
h_{3}^{\prime} & =\nu\left(h_{1} h_{2}-5 e_{1} e_{2}\right), \\
e_{1}^{\prime} & =-\frac{4+\nu}{2} h_{2} e_{3}+\frac{5}{2} \nu h_{3} e_{2}+\frac{n \nu}{\Omega_{0}} e_{2}, \\
e_{2}^{\prime} & =\frac{4-\nu}{2} h_{1} e_{3}+\frac{5}{2} \nu h_{3} e_{1}-\frac{n \nu}{\Omega_{0}} e_{1}, \\
e_{3}^{\prime} & =\frac{1-4 \nu}{2} h_{1} e_{2}-\frac{1+4 \nu}{2} h_{2} e_{1} .
\end{aligned}
$$

Substituting $\nu=0$, the readers may recover the correct form of the Galactic disc tide equations published in $[3,4]$. Equations (72)-(77) admit three integrals of motion: apart from the usual conservation of the time-independent Hamiltonian $\mathcal{K}=$ const, two geometrical constraints

$$
\boldsymbol{h} \cdot \boldsymbol{e}=0, \quad h^{2}+e^{2}=1,
$$

are respected thanks to the properties of the Lie-Poisson bracket (65). Indeed, both quadratic forms are the Casimir functions of our bracket, i.e.

$$
(\boldsymbol{h} \cdot \boldsymbol{e} ; f)=\left(h^{2}+e^{2} ; f\right)=0,
$$

for any function $f$, hence in particular for $f=\mathcal{K}$.

\section{Lie-Poisson splitting method}

The Hamiltonian $\mathcal{K}$ can be split into a sum of three non-commuting terms

$$
\begin{aligned}
\mathcal{K} & =\mathcal{K}_{1}+\mathcal{K}_{2}+\mathcal{K}_{3}, \\
\mathcal{K}_{1} & =\frac{5}{4} \nu e_{1}^{2}-\frac{1+\nu}{4} h_{1}^{2}, \\
\mathcal{K}_{2} & =-\frac{5}{4} \nu e_{2}^{2}-\frac{1-\nu}{4} h_{2}^{2}, \\
\mathcal{K}_{3} & =-\frac{5}{4} e_{3}^{2}+\frac{n \nu}{\Omega_{0}} h_{3} .
\end{aligned}
$$


Each of the terms $\mathcal{K}_{i}$ is in turn a sum of two components that commute, because it can be easily verified that $\left(e_{j} ; h_{j}\right)=0$ for all $j \in\{1,2,3\}$. In these circumstances, we can approximate the real solution

$$
\boldsymbol{v}(\tau)=\exp (\tau L) \boldsymbol{v}(0),
$$

where $L f \equiv(f ; \mathcal{K})$, using a composition of maps

$$
\Psi_{i, \tau}: \quad \boldsymbol{v}(0) \rightarrow \boldsymbol{v}(\tau)=\exp \left(\tau L_{i}\right) \boldsymbol{v}(0),
$$

where $L_{i} f \equiv\left(f ; \mathcal{K}_{i}\right)$ for $i=1,2,3$. Each $\Psi_{i, \tau}$ is in turn a composition of two maps

$$
\Psi_{i, \tau}=E_{i, \tau} \circ H_{i, \tau}=H_{i, \tau} \circ E_{i, \tau},
$$

generated by the $e_{i}$ and $h_{i}$ related terms of $\mathcal{K}_{i}$.

\section{The contribution of $\mathcal{K}_{1}$}

The two terms of $\mathcal{K}_{1}$ generate equations of motion

$$
\boldsymbol{v}^{\prime}=\left(\boldsymbol{v} ; \frac{5}{4} \nu e_{1}^{2}\right)=\frac{5}{2} e_{1} \nu\left(\begin{array}{cc}
\mathbf{0} & \mathbf{Y}_{1} \\
\mathbf{Y}_{1} & \mathbf{0}
\end{array}\right) \boldsymbol{v}
$$

and

$$
\boldsymbol{v}^{\prime}=\left(\boldsymbol{v} ;-\frac{1}{4}(1+\nu) h_{1}^{2}\right)=-\frac{1}{2} h_{1}(1+\nu)\left(\begin{array}{cc}
\mathbf{0} & \mathbf{Y}_{1} \\
\mathbf{Y}_{1} & \mathbf{0}
\end{array}\right) \boldsymbol{v}
$$

where

$$
\mathbf{Y}_{1}=\left(\begin{array}{ccc}
0 & 0 & 0 \\
0 & 0 & -1 \\
0 & 1 & 0
\end{array}\right)
$$

The composition of these two maps results in

$$
\Psi_{1, \tau}: \boldsymbol{v} \rightarrow\left(\begin{array}{ll}
\mathbf{M}_{1} & \mathbf{N}_{1} \\
\mathbf{N}_{1} & \mathbf{M}_{1}
\end{array}\right) \boldsymbol{v}
$$

where

$$
\begin{aligned}
& \mathbf{M}_{1}=\left(\begin{array}{ccc}
1 & 0 & 0 \\
0 & c_{11} c_{12} & -c_{11} s_{12} \\
0 & c_{11} s_{12} & c_{11} c_{12}
\end{array}\right), \quad \mathbf{N}_{1}=\left(\begin{array}{ccc}
0 & 0 & 0 \\
0 & s_{11} s_{12} & s_{11} c_{12} \\
0 & -s_{11} c_{12} & s_{11} s_{12}
\end{array}\right) \\
& \psi_{11}=\frac{5}{2} e_{1} \nu \tau, \quad \text { and } \quad \psi_{12}=\frac{1}{2}(1+\nu) h_{1} \tau,
\end{aligned}
$$

introducing

$$
c_{i j}=\cos \psi_{i j}, \quad s_{i j}=\sin \psi_{i j} .
$$




\section{The contribution of $\mathcal{K}_{2}$}

The equations of motion derived from the two terms of $\mathcal{K}_{2}$ are

$$
\boldsymbol{v}^{\prime}=\left(\boldsymbol{v} ;-\frac{5}{4} \nu e_{2}^{2}\right)=\frac{5}{2} \nu e_{2}\left(\begin{array}{cc}
\mathbf{0} & \mathbf{Y}_{2} \\
\mathbf{Y}_{2} & \mathbf{0}
\end{array}\right) \boldsymbol{v},
$$

and

$$
\boldsymbol{v}^{\prime}=\left(\boldsymbol{v} ;-\frac{1}{4}(1-\nu) h_{2}^{2}\right)=\frac{1}{2}(1-\nu) h_{2}\left(\begin{array}{cc}
\mathbf{0} & \mathbf{Y}_{2} \\
\mathbf{Y}_{2} & \mathbf{0}
\end{array}\right) \boldsymbol{v}
$$

where

$$
\mathbf{Y}_{2}=\left(\begin{array}{ccc}
0 & 0 & 1 \\
0 & 0 & 0 \\
-1 & 0 & 0
\end{array}\right)
$$

Composing the two maps we obtain:

$$
\Psi_{2, \tau}: \boldsymbol{v} \rightarrow\left(\begin{array}{ll}
\mathbf{M}_{2} & \mathbf{N}_{2} \\
\mathbf{N}_{2} & \mathbf{M}_{2}
\end{array}\right) \boldsymbol{v}
$$

where

$$
\begin{aligned}
& \mathbf{M}_{2}=\left(\begin{array}{ccc}
c_{21} c_{22} & 0 & -c_{21} s_{22} \\
0 & 1 & 0 \\
c_{21} s_{22} & 0 & c_{21} c_{22}
\end{array}\right), \quad \mathbf{N}_{2}=\left(\begin{array}{cccc}
s_{21} s_{22} & 0 & c_{22} s_{21} \\
0 & 0 & 0 \\
-c_{22} s_{21} & 0 & s_{21} s_{22}
\end{array}\right) \\
& \psi_{21}=\frac{5}{2} \nu e_{2} \tau, \quad \text { and } \quad \psi_{22}=-\frac{h_{2}(1-\nu)}{2} \tau .
\end{aligned}
$$

\section{The contribution of $\mathcal{K}_{3}$}

The equations of motion derived from the two terms of $\mathcal{K}_{3}$ are

$$
\boldsymbol{v}^{\prime}=\left(\boldsymbol{v} ;-\frac{5}{4} e_{3}^{2}\right)=\frac{5}{2} e_{3}\left(\begin{array}{cc}
\mathbf{0} & \mathbf{Y}_{3} \\
\mathbf{Y}_{3} & \mathbf{0}
\end{array}\right) \boldsymbol{v}
$$

and

$$
\boldsymbol{v}^{\prime}=\left(\boldsymbol{v} ; h_{3} n \nu \Omega_{0}^{-1}\right)=-\frac{n \nu}{\Omega_{0}}\left(\begin{array}{cc}
\mathbf{0} & \mathbf{Y}_{3} \\
\mathbf{Y}_{3} & \mathbf{0}
\end{array}\right) \boldsymbol{v}
$$

where

$$
\mathbf{Y}_{3}=\left(\begin{array}{ccc}
0 & -1 & 0 \\
1 & 0 & 0 \\
0 & 0 & 0
\end{array}\right)
$$

Composing the two maps we obtain

$$
\Psi_{3, \tau}: \boldsymbol{v} \rightarrow\left(\begin{array}{ll}
\mathbf{M}_{3} & \mathbf{N}_{3} \\
\mathbf{N}_{3} & \mathbf{M}_{3}
\end{array}\right) \boldsymbol{v}
$$


where

$$
\begin{gathered}
\mathbf{M}_{3}=\left(\begin{array}{ccc}
c_{31} c_{32} & c_{31} s_{32} & 0 \\
-c_{31} s_{32} & c_{31} c_{32} & 0 \\
0 & 0 & 1
\end{array}\right), \quad \mathbf{N}_{3}=\left(\begin{array}{ccc}
s_{31} s_{32} & -c_{32} s_{31} & 0 \\
c_{32} s_{31} & s_{31} s_{32} & 0 \\
0 & 0 & 0
\end{array}\right) \\
\psi_{31}=\frac{5}{2} e_{3} \tau, \quad \text { and } \quad \psi_{32}=\frac{n \nu}{\Omega_{0}} \tau .
\end{gathered}
$$

\subsection{The Lie-Poisson method of order 2}

The composition methods of [11] cannot be used for our Lie-Poisson splitting method, because the Hamiltonian function has been partitioned into three terms. Moreover, none of the terms can be qualified as a small perturbation. In these circumstances, the principal building block can be a 'generalised leapfrog'

$$
\Psi_{\Delta}=\Psi_{1, \Delta / 2} \circ \Psi_{2, \Delta / 2} \circ \Psi_{3, \Delta} \circ \Psi_{2, \Delta / 2} \circ \Psi_{1, \Delta / 2} .
$$

This Lie-Poisson method, called LPV2, is a second order method with a local truncation error proportional to the cube of the step-size $\Delta^{3}$. Although we use LPV2 as a final product in this paper, it can be used as a building block for higher-order methods. A collection of appropriate composition rules can be found in [13].

In practice, the step size $\Delta$ will be set equal to one orbital period.

\section{Comparisons between the different models}

In order to compare the reliability and speed of the integrators we performed the following experiment: 400000 sets of initial orbital elements were randomly chosen in a specified range, under the condition that their respective distribution is uniform, i.e.:

- the initial semi-major axes in the range $3000 \leq a_{0} \leq 10^{5} \mathrm{AU}$, with distribution uniform in $\log _{10} a_{0}$;

- the initial eccentricity in the range $0 \leq e_{0} \leq 0.9999$, with a uniform distribution;

- the initial inclination $i_{0}$ such that $-1 \leq \cos i_{0} \leq 1$, with a uniform distribution;

- the initial argument of the perihelion, the longitude of the ascending node, and the initial mean anomaly (where needed) in the range from 0 to $2 \pi$, with a uniform distribution.

Using this set of elements, we integrated the equations over one cometary period using LARKS, LPV2, MAPP and compared the results with those obtained with the Cartesian model. 
For the stopping time in LARKS, the method described at the end of Section 2.2 until $\left|T_{f}-t\right|<10^{-3}$ yr was used.

The relative error in the comet position $E_{\mathrm{p}}$ was defined as

$$
E_{\mathrm{p}}=\left|\frac{q_{\mathrm{mod}}-q_{\mathrm{R}}}{q_{0}}\right| \text {, }
$$

where $q_{\mathrm{mod}}$, and $q_{\mathrm{R}}$ denote the value of the perihelion distance at the end of the integration of one period computed by the tested integrator and by the RADAU respectively, and $q_{0}$ is the initial value of the perihelion distance.

Then, the $e_{0}-\log _{10} a_{0}$ plane is divided into $60 \times 70$ cells. In each cell we record the maximum value $E_{\max }$ reached by the error $E_{\mathrm{p}}$ for the initial conditions belonging to the cell.

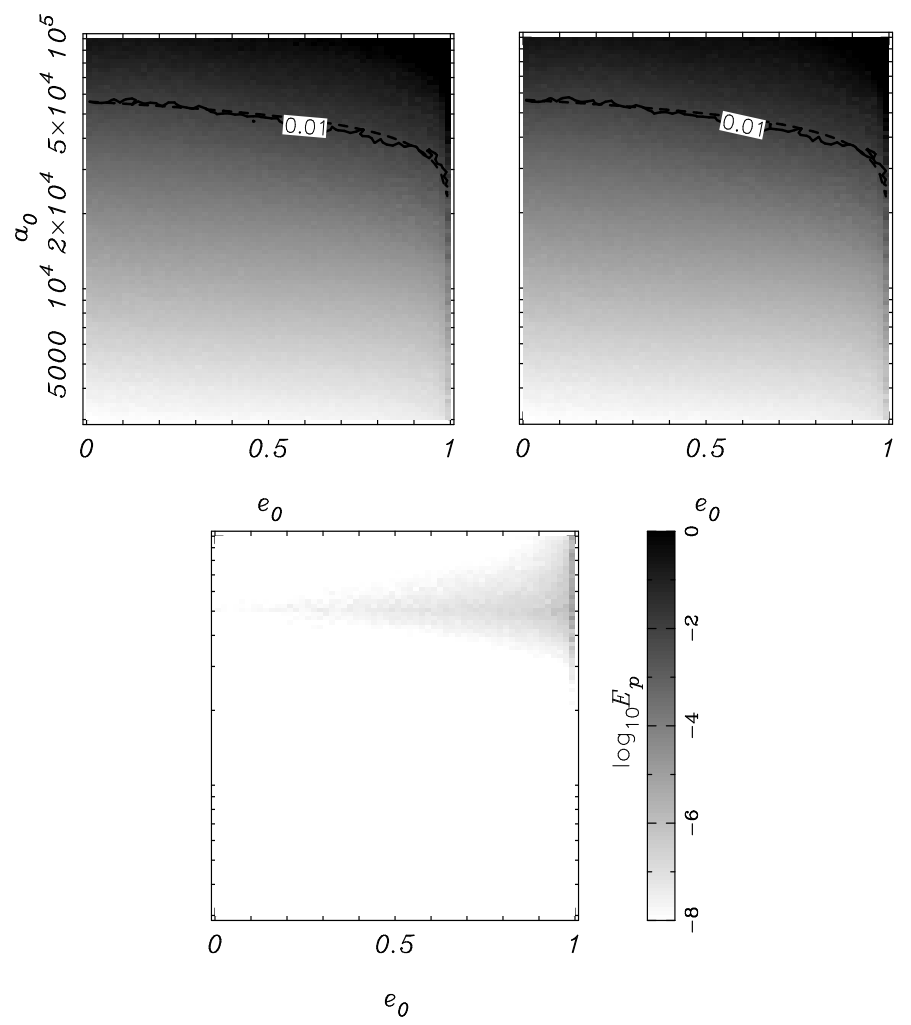

Fig. 1. Maximium error $E_{\mathrm{p}}$ (see Eq. 107) in each cell of the $e_{0}-a_{0}$ plane for the models MAPP (top left), LPV2 (top right), and LARKS (bottom). The solid line curves correspond to $E_{\mathrm{p}}=0.01$ and the dotted curves are the best fits of the level curves. 
The results obtained for the three models are shown in Fig. 1. The MAPP and the LPV2 models, both used with a step size equal to the unperturbed Keplerian period, are equivalent as far as the accuracy is concerned. Indeed, the best analytical fit of the level curve $E_{\mathrm{p}}=0.01$ is given by:

$$
a_{c}=10^{4.748 \pm 0.004}(1-e)^{0.182 \pm 0.006}
$$

for the MAPP model and,

$$
a_{c}=10^{4.751 \pm 0.003}(1-e)^{0.185 \pm 0.005}
$$

for the LPV2 model. These two equations may be considered as identical within the error bounds of the exponents.

For both models the error is essentially due to the averaging of the equations of motion with respect to the mean anomaly. Conversely, the LARKS method is highly reliable in the whole phase space domain under study, since the error never exceed 0.01 . The effect of the time step selection rule (42) is clearly visible above $a_{0}=50000 \mathrm{AU}$; the reliability of LARKS is almost conserved when $a_{0}$ increases.

Speaking about the computation times required to perform all the integrations, the MAPP, LPV2 and LARKS needed 5.5, 1.8 and 99 seconds, whereas the RA15 integration took 1820 seconds. That is, LPV2 is 3 times faster than MAPP, and almost 40 times faster than LARKS, and LARKS is almost 20 times faster than RA15.

\section{Hybrid integrators}

\subsection{Definition}

In order to have the best compromise between velocity and precision, one can consider hybrid models which use the fastest accurate model according to the values of the cometary eccentricity and semi-major axis.

In [9] the hybrid model MAPP + RADAU, was introduced and applied to reproduce the effects of the galactic tide on the dynamics of $10^{6}$ comets over 5 Gyr. This hybrid model was such that MAPP was used below the analytical fit of the $E_{\mathrm{p}}=0.01$ level curve given by Eq. (108), otherwise RADAU was used.

This hybrid model became obsolete since [5] where LPV2 and LARKS have been introduced. Indeed, the hybrid model LPV2 + LARKS, where LPV2 is used below the analytical fit of the $E_{\mathrm{p}}=0.01$ level curve given by Eq. (109), is much faster than MAPP + RADAU, and has the same accuracy. In the special case of a galactic potential such that $A \neq-B$, i.e. $\mathcal{G}_{2} \neq-\mathcal{G}_{1}$, LARKS is easily generalisable whereas LPV2 is not. Thus in such a case the hybrid model MAPP + LARKS may be used. 
Let us consider the integration of a comet with a hybrid model, say LARKS + LPV2. The oscillation of the eccentricity with time may lead to repeating shifts between the use of LARKS and the use of LPV2. However, when LARKS is used the semi-major axis oscillates with a period equal to the orbital period of the comet (this may be easily understood from the fact that in the Hamiltonian formalism, the mean anomaly and $L=\sqrt{\mu a}$ are conjugate coordinates), whereas it is a constant of motion when LPV2 is used. Indeed, for the LPV2 model the mean-anomaly cancels out.

Since LPV2 is applied for an integer number of orbital periods, one may just record the value of the mean-anomaly at the beginning of a sequence where LPV2 is used, and restore the mean-anomaly value at the end of the sequence. Consequently, when one shifts from LPV2 to LARKS, the memory of the orginal orbit is conserved, as far as the averaging is neglected.

However, a shift from LARKS to LPV2 occurs for an arbitrary value of the mean anomaly, thus it occurs for an arbitrary value of the semi-major axis in the interval of its oscillations. Consequently, from one such shift to another one, the LPV2 model will be applied to different averaged orbits since the semi-major axis is different. After many shifts, a drift on the semi-major axis value may be observed.

An easy way to remove this drift is to allow the shift between LARKS and LPV2 only when the comet is exactly at its perihelion. Indeed, in this way, the shift occurs always at a precise time of the semi-major axis oscillation, thus the memory of the real orbit may be conserved when many shifts from LARKS to LPV2 are performed.

\section{Conclusion}

Different models of the galactic tide have been presented. The first one, called LARKS, is a symplectic integrator which uses the Kuustanheimo-Stiefel (KS) transformation to regularise the equations of motion. This model turns out to be reliable over the whole phase space and almost 20 times faster than a non symplectic integrator using Cartesian coordinates (RADAU). The two other models are based on the averaging of the equations of motion with respect to the mean anomaly. One, which is called MAPP, uses the Taylor development at order three of the solutions of the averaged equations of motion. In this case the equations are written using the Hamiltonian formalism with two different sets of canonical variables according to the value of the eccentricity. The second averaged model, called LPV2, considers the equations of motion using the normalised Laplace and angular momentum vectors. Then a LiePoisson integrator of order 2 is used by splitting the Hamiltonian in three parts.

As regards the accuracy, these two models are equivalent, but they are reliable only in a limited domain of the phase space. These models are both faster than LARKS, but LPV2 is 3 times faster than MAPP, and 40 times 
faster than LARKS. The main advantage of MAPP is that it is more didactic. MAPP is also more general since it may consider any kind of radial component of the tide - but with the assumption that the tide is axi-symmetric -, whereas LPV2 requires $\mathcal{G}_{2}=-\mathcal{G}_{1}$.

The best hybrid model can be defined as a combination between the use of LPV2 and LARKS according to the value of the cometary eccentricity and semi-major axis. For instance, if one wants a confidence level of $1 \%$ on the perihelion distance variation over one cometary period, one may use the analytical equation given by

$$
a_{c}=10^{4.751}(1-e)^{0.185},
$$

to define the upper frontier of the domain where LPV2 may be used.

If one wants a confidence level of $0.1 \%$ one may consider the value:

$$
a_{c}=10^{4.570}(1-e)^{0.176} \text {, }
$$

as the upper limit of the domain where LPV2 may be used. However, the use of Eq. (111) will slow down the integrations since it reduces the domain of application of LPV2. The above hybrid model may be used for any long term simulations of the Oort cloud comets dynamics under the effects of the galactic tide.

\section{References}

1. R. Brasser: MNRAS 324, 1109 (2001)

2. S. Breiter: CeMDA 71, 229 (1998)

3. S. Breiter, R. Ratajczak: MNRAS 364, 1222 (2005)

4. S.Breiter, R. Ratajczak: MNRAS 367, 1808 (2006)

5. S. Breiter, M. Fouchard, R. Ratajczak: MNRAS (submitted)

6. A. Deprit, A. Elipe, S. Ferrer: CeMDA 58, 151 (1994)

7. E. Everhart: in: Dynamics of Comets: Their Origin and Evolution, eds. A. Carusi, G.B. Valsecchi, Reidel, Dordrecht (1985), p. 185

8. M. Fouchard, Ch. Froeschlé, J.J. Matese, G.B. Valsecchi: CeMDA 93, 231 (2005)

9. M. Fouchard, Ch. Froeschlé, G.B. Valsecchi, H. Rickman: CeMDA 95, 299 (2006)

10. J. Heisler, S. Tremaine: Icarus 65, 13 (1986)

11. J. Laskar, P. Robutel: CeMDA 80, 39 (2001)

12. H. Levison, L. Dones, M.J. Duncan: AJ 121, 2253 (2001)

13. R.I. McLachlan, G.R.W. Quispel: Acta Numerica, 11, 341 (2002)

14. J.J. Matese, P.G. Whitman: Icarus 82, 389 (1989)

15. J.J. Matese, P.G. Whitman: CeMDA 54, 13 (1992)

16. E.L. Stiefel, G. Scheifele: Linear and Regular Celestial Mechanics, SpringerVerlag, Berlin-New York (1971)

17. Tommei, G.: CeMDA 94, 176 (2006)

18. Tremaine, S.: CeMDA 79, 231 (2001)

19. J. Wisdom, M. Holman: AJ 104, 2022 (1992) 